CUBO A Mathematical Journal Vol.13, Noㅇ, (59-72). June 2011

\title{
On $\lambda$ strong homogeneity existence for cofinality logic
}

\author{
SAHARON SHELAH 1 \\ Einstein Institute of Mathematics, \\ The Hebrew University of Jerusalem, \\ Jerusalem, 91904, Israel. \\ email: shelah@math.huji.ac.il \\ url: http://shelah.logic.at
}

\begin{abstract}
Let $C \subset_{\neq}$Reg be a non-empty class (of regular cardinals). Then the logic $\mathbb{L}\left(\mathrm{Q}_{C}^{\text {cf }}\right)$ has additional nice properties: it has the homogeneous model existence property.
\end{abstract}

\section{RESUMEN}

Sea $C \subset \neq$ Reg una clase no vacía (de cardinales regulares). Entonces la lógica $\mathbb{L}\left(Q_{C}^{c f}\right)$ tiene propiedades adicionales: Esta tiene la propiedad de modelo existencia homogénea.

Keywords and phrases: Model theory, soft model theory, cofinality quantifier. Mathematics Subject Classification: 03C95, 03 C80.

\footnotetext{
${ }^{1}$ The author thanks Alice Leonhardt for the beautiful typing. The author would like to thank the Israel Science Foundation for partial support of this research (Grants No. 451/99 and 710/07). Publication 750.
} 


\section{Introduction}

We deal with logics gotten by strengthening of first order logic by generalized quantifiers, in particular compact ones. We continue [Sh:199] (and [Sh:43])

A natural quantifier is the cofinality quantifier, $Q_{\leq \lambda}^{\text {cf }}\left(\right.$ or $Q_{C}^{\text {cf }}$ ), introduced in Sh:43] as the first example of compact logic (stronger than first order logic, of course). Recall that the "uncountably many $x$ 's" quantifier $\mathrm{Q}_{\geq \boldsymbol{\aleph}_{1}}^{\text {card }}$, is $\boldsymbol{\aleph}_{0}$-compact but not compact. Also note that $\mathbb{L}\left(\mathrm{Q}_{\leq \lambda}^{\mathrm{cf}}\right)$ is a very nice logic, e.g. with a nice axiomatization (in particular finitely many schemes) like the one of $\mathbb{L}\left(Q_{\geq \mathbf{\Sigma}_{1}}^{\text {card }}\right)$ of Keisler. By Sh:199], e.g. for $\lambda=2^{\boldsymbol{\aleph}_{0}}$, its Beth closure is compact, giving the first compact logic with the Beth property (i.e. implicit definition implies explicit definition).

Earlier there were indications that having the Beth property is rare for such logic, see e.g. in Makowsky [Mak85]. A weaker version of the Beth property is the weak Beth property dealing with implicit definition which always works; H. Friedman claim that historically this was the question. Mekler-Shelah MkSh:166] prove that at least consistently, $\mathbb{L}\left(Q_{\geq \mathbb{\aleph}_{1}}^{\text {card }}\right)$ satisfies the weak Beth property. Väänänen in the mid nineties motivated by the result of Mekler-Shelah [MkSh:166] asked whether we can find a parallel proof for $\mathbb{L}\left(Q_{\leq \lambda}^{\text {cf }}\right)$ in $\mathrm{ZFC}$.

A natural property for a logic $\mathcal{L}$ is

Definition 1. A logic $\mathcal{L}$ has the (strong) homogeneous model existence property when every theory $\mathrm{T} \subseteq \mathcal{L}(\tau)$, (so has a model) has a strongly $\left(\mathcal{L}, \boldsymbol{\aleph}_{0}\right)$-homogeneous model $M$, so $\tau_{M}=\tau$ and $M$ is a model of $T$ and $M$ satisfies: if $\bar{a}, \bar{b} \in{ }^{\omega>} M$ realize the same $\mathcal{L}(\tau)$-type in $M$ then there is an automorphism of $M$ mapping $\bar{a}$ to $\bar{b}$.

This property was introduced in Sh:199] being natural and also as it helps to investigate the weak Beth property.

In $\S 1$ we prove that $\mathbb{L}\left(Q_{C}^{\text {cf }}\right)$ has the strongly $\boldsymbol{\aleph}_{0}$-saturated model existence property. The situation concerning the weak Beth property is not clear.

Question 2. 1) Does the logic $\mathbb{L}\left(\mathrm{Q}_{\mathrm{C}}^{\mathrm{cf}}\right)$ have the weak Beth property?

2) Does the logic $\mathbb{L}\left(Q_{\leq \lambda_{1}}^{c f}, Q_{\leq \lambda_{2}}^{c f}\right)$ has the homogeneous model existence property?

The first version of this work was done in 1996.

Notation 3. 1) $\tau$ denotes a vocabulary, $\mathcal{L}$ a logic, $\mathcal{L}(\tau)$ the language for the logic $\mathcal{L}$ and the vocabulary $\tau$.

2) Let $\mathbb{L}$ be first order logic, $\mathbb{L}\left(Q_{*}\right)$ be first order logic when we add the quantifier $Q_{*}$.

3) For a model $M$ and ultrafilter $D$ on a cardinal $\lambda$, let $M^{\lambda} / D$ be the ultrapower and $\mathbf{j}_{M, D}=\mathbf{j}_{M, D}^{\lambda}$ be the canonical embedding of $M$ into $M^{\lambda} / D$; of course, we can replace $\lambda$ by any set.

4) Let LST (theorem/argument) stand for Löwenheim-Skolem-Tarski (on existence of elementary submodels). 
Concerning 1, more generally

Definition 4. 1) $M$ is strongly $(\mathcal{L}, \theta)$-saturated (in $\mathcal{L}=\mathbb{L}$ we may write just $\theta$ ) when

(a) it is $\theta$-saturated (i.e. every set of $\mathcal{L}\left(\tau_{M}\right)$-formulas with $<\theta$ parameters from $M$ and $<\theta$ free variables which is finitely satisfiable in $M$ is realized in $M$

(b) if $\zeta<\theta$ and $\bar{a}, \bar{b} \in \zeta M$ realize the same $\mathcal{L}\left(\tau_{M}\right)$-type in $M$, then some automorphism of $M$ maps $\bar{a}$ to $\bar{b}$.

2) $M$ is a strongly sequence $(\mathcal{L}, \theta)$-homogeneous when clause (b) above holds.

3) $M$ is sequence $(\Delta, \kappa)$-homogeneous when: $\Delta \subseteq \mathcal{L}\left(\tau_{M}\right)$ and if $\zeta<\kappa, \bar{a} \in \zeta^{\zeta} M, \bar{b} \in \zeta M$ and $\operatorname{tp}_{\Delta}(\bar{a}, \emptyset, M)=\operatorname{tp}_{\Delta}(\bar{b}, \emptyset, M)$ then for every $c \in M$ for some $d \in M$ we have $\operatorname{tp}_{\Delta}\left(\bar{b}^{\wedge}\langle d\rangle, \emptyset, M\right)=$ $\operatorname{tp}_{\Delta}(\overline{\mathrm{a}} \wedge\langle\mathrm{c}\rangle, M)$.

3A) $\Sigma_{1}(\tau)$ is the set of formulas of the form $\varphi(\bar{x})=(\exists \bar{y}) \vartheta(x, \bar{y})$ where $\vartheta(\bar{x}, \bar{y})$ is quantifier free first order formula in the vocabulary $\tau$.

4) We may omit "sequence".

Definition 5. 1) The logic $\mathcal{L}$ has "the strong $\kappa$-homogeneous existence property" when every theory $\mathrm{T} \subseteq \mathcal{L}\left(\tau_{1}\right)$ has a strongly $(\mathcal{L}, \mathrm{k})$-homogeneous model.

2) Similarly "the strong K-saturated existence property", etc.

\section{On strongly saturated models}

We prove that any theory in $\mathbb{L}\left(Q_{C}^{\text {cf }}\right)$ has strongly $\left(\mathbb{L}\left(Q_{C}^{\text {cf }}\right), \theta\right)$-saturated model when $C \backslash \theta \notin$ $\{\emptyset, \operatorname{Reg} \backslash \theta\}$ of course.

Definition 6. Let $\iota \in\{1,2\}$ and $C$ be a class of regular cardinals such that $C \neq \emptyset$, Reg.

1) The quantifier $Q_{C}^{\mathrm{cf}(\iota)}$ is defined as follows:

syntactically: it bounds two variables, i.e. we can form $\left(Q_{C}^{\mathrm{cf}(\iota)} x, y\right) \varphi$, with its set of free variables being defined as $\mathrm{F} \operatorname{Var}(\varphi) \backslash\{x, y\}$.

semantically: $M \models\left(Q_{C}^{c f(\iota)} x, y\right) \varphi(x, y, \bar{a})$ iff $(a)+(b)$ holds where

(a) relevancy demand:

the case $\iota=1$ : the formula $\varphi(-,-; \overline{\mathbf{a}})^{M}$ define in $M$ a linear order with no last element called $\leq_{M, \bar{a}}^{\varphi}$ on the non-empty set $\operatorname{Dom}\left(\leq_{M}^{\varphi}, \bar{a}\right)=\{b \in M: M \models(\exists y)(\varphi(b, y ; \bar{a})\}$

The case $\iota=2$ : similarly but $\leq_{M, \overline{\mathrm{a}}}^{\varphi}$ is a quasi linear order on its domain 
(b) the actual demand: $\leq_{M}^{\varphi}, \overline{\mathrm{a}}$ has cofinality $\operatorname{cf}\left(\leq_{\mathcal{M}, \overline{\mathrm{a}}}^{\varphi}\right)$, (necessarily an infinite regular cardinal) which belongs to $\mathrm{C}$.

Convention 7. 1) Writing $Q_{C}^{c f}$ we mean that this holds for $Q_{C}^{c f(\iota)}$ for $\iota=1$ and for $\iota=2$.

2) Let $\iota$-order mean order when $\iota=1$ and quasi order when $\iota=2$; but when we are using $Q_{C}^{\operatorname{cf}(\iota)}$ then order means l-order.

Definition 8. 1) As $\left\{\psi \in \mathbb{L}\left(Q_{C}^{c f}\right): \psi\right.$ has a model $\}$ does not depend on $C$ (and is compact, see Sh:43] ) we may use the formal quantifier $\mathrm{Q}_{\mathrm{cf}}$, so the syntex is determined but not the semantics, i.e. the satisfaction relation $\models$. We shall write $M \models_{\mathrm{C}} \psi$ or $M \models_{\mathrm{C}} \mathrm{T}$ for the interpretation of $\mathrm{Q}_{\mathrm{cf}}$ as $\mathrm{Q}_{\mathrm{C}}^{\mathrm{cf}}$, but also can say "T $\subseteq \mathbb{L}\left(\mathrm{Q}_{\mathrm{cf}}\right)(\tau)$ has model/is consistent".

2) If $C$ is clear from the context, then $Q_{\ell}^{\text {cf }}$ stands for $Q_{C}^{c f}$ if $\ell=1$ and $Q_{\operatorname{Reg} \backslash C}^{\text {cf }}$ if $\ell=0$.

Convention 9. 1) $\mathrm{T}^{*}$ is a complete (consistent $\equiv$ has models) theory in $\mathbb{L}\left(\mathrm{Q}_{\mathrm{cf}}\right)$ which is closed under definitions i.e. every formula $\varphi=\varphi(\bar{x})$ is equivalent to a predicate $\mathrm{P}_{\varphi}(\bar{x})$ so $\mathrm{P}_{\varphi} \in \tau\left(\mathrm{T}^{*}\right)$, i.e. $\mathrm{T}^{*} \vdash(\forall \bar{z})\left[\varphi(\bar{z}) \equiv \mathrm{P}_{\varphi}(\bar{z})\right]$.

2) Let $T=T^{*} \cap$ (first order logic), i.e. $T=T^{*} \cap \mathbb{L}\left(\tau_{T^{*}}\right)$, it is a complete first order theory.

3) $\mathrm{C} \subseteq$ Reg, we let $\mathrm{C}_{1}=\mathrm{C}$ and $\mathrm{C}_{0}=\operatorname{Reg} \backslash \mathrm{C}$, both non-empty.

Theorem 10. Assume $\chi=\operatorname{cf}(\chi), \mu=\mu^{<\theta} \geq 2^{|\mathrm{T}|}+\chi+\kappa, \theta \leq \lambda, \operatorname{cf}(\theta) \leq \min \{\chi, \kappa\}, \chi \neq \kappa=\operatorname{cf}(\kappa)$ and

$$
\mu_{\ell}= \begin{cases}x & \ell=0 \\ \kappa & \ell=1\end{cases}
$$

Then there is a $\tau(T)$-model $M$ such that

(a) $M \models T,\|M\|=\mu$ and $M$ is $\theta$-saturated

(b) if $\varphi(\bar{z})=\left(Q_{\ell}^{c f}\right) \psi(x, y ; \bar{z})$ then: $M \models P_{\varphi(\bar{x})}[\bar{a}]$ iff $\varphi(y, z ; \bar{a})$ define in $M$ a linear order with no last element and cofinality $\mu_{\ell}$

(c) $M$ is strongly $\theta$-saturated model of $\mathrm{T}^{*}$.

Remark 11. 1) We can now change $\chi, \kappa, \mu$ and $\|M\|$ by LST. Almost till the end instead $\mu \geq$ $2^{|\mathrm{T}|}+\chi+\kappa$ just $\mu \geq|\mathrm{T}|+\chi+\kappa$ suffice. The proof is broken to a series of definitions and claims. The " $\geq 2^{|T|}$ " is necessary for $\boldsymbol{x}_{0}$-saturativity.

3) We can assume $\mathbf{V}$ satisfies GCH high enough and then use LST. So $\mu^{+}=2^{\mu}$ below is not a real burden.

\footnotetext{
${ }^{2}$ as $\mathrm{T}^{*}$ has elimination of quantifiers, doing it for $\mathbb{L}\left(\mathrm{Q}_{\mathrm{C}}^{\mathrm{cf}}\right)$ or for $\mathbb{L}$ is the same
} 
Definition 12. 0) ModT is the class of models of T.

1)

(a) $K=\left\{(M, N): M \prec N\right.$ are from $\left.\operatorname{Mod}_{T}\right\}$

(b) $K_{\alpha}=\left\{\bar{M}: \bar{M}=\left\langle M_{i}: i<\alpha\right\rangle\right.$ satisfies $M_{i} \in \operatorname{Mod}_{\mathrm{T}}$ and $\left.i<j \Rightarrow M_{i} \prec M_{j}\right\}$ (so $K=K_{2}$ )

(c) $K_{\mu}^{\alpha}=\left\{\bar{M} \in K_{\alpha}:\left\|M_{i}\right\| \leq \mu\right.$ for $\left.i<\alpha\right\}$, but then we (naturally) assume $\alpha<\mu^{+}$

(d) let $\tau_{\alpha}=\tau_{\mathrm{T}} \cup\left\{\mathrm{P}_{\beta}: \beta<\alpha\right\} \cup\left\{\mathrm{R}_{\varphi(x, y, \bar{z}), \beta}: \varphi(x, y, \bar{z}) \in \mathbb{L}\left(\tau_{\mathrm{T}}\right), \beta<\alpha\right\}$, each $\mathrm{P}_{\beta}$ a unary predicate and each $R_{\varphi(x, y, \bar{z}), \beta}$ is an $(\lg (\bar{z})+1)$-place predicate and no incidental identification (so $\mathrm{P}_{\alpha} \notin \tau$, etc.)

(e) for $\bar{M} \in \mathrm{K}_{\alpha}$ let $\mathbf{m}(\bar{M})$ be the $\tau_{\alpha}$-model $M$ with

- universe $\cup\left\{M_{\beta}: \beta<\alpha\right\}$

- $M \mid \tau_{\mathrm{T}}=\cup\left\{M_{\beta}: \beta<\alpha\right\}$

- $P_{\beta}^{M}=M_{\beta}$

- $\mathrm{R}_{\varphi(x, y, \bar{z}), \beta}^{M}=\left\{\langle c\rangle^{\wedge} \bar{a}: \varphi(x, y, \bar{a})\right.$ a linear order, $\bar{a} \in{ }^{\ell g(\bar{z})}\left(P_{\beta}^{M}\right)$ such that $M \models P_{\left(Q_{0}^{c f} x, y\right) \varphi(x, y, \bar{z})}[\bar{a}]$ and $\mathrm{c} \in \operatorname{Dom}\left(\leq_{M}^{\varphi}, \bar{a}\right)$ and $\left.\left[\mathrm{b} \in \operatorname{Dom}\left(\leq_{M}^{\varphi}, \bar{a}\right) \cap P_{\beta}^{M} \Rightarrow \mathrm{b} \leq_{M}^{\varphi}, \bar{a} \mathrm{c}\right]\right\}$

(f) let $\mathbf{m}_{0}(\bar{M})$ be the $\tau$-model $\cup\left\{M_{\beta}: \beta<\alpha\right\}$ so $\mathbf{m}_{0}(\bar{M})=\mathbf{m}(\bar{M})\lceil\tau$.

2) Assume $\left(M^{\ell}, N^{\ell}\right) \in K$ for $\ell=1,2$ let $\left(M^{1}, N^{1}\right) \leq\left(M^{2}, N^{2}\right)$ mean that clauses (a),(b),(c) below hold and let $\left(M^{1}, N^{1}\right) \leq_{K}\left(M^{2}, N^{2}\right)$ mean that in addition clause (d) below holds, where:

(a) $M^{1} \prec M^{2}$

(b) $M^{2} \cap N^{1}=M^{1}$

(c) $\mathrm{N}^{1} \prec \mathrm{N}^{2}$

(d) if $M^{1} \models P_{\left(Q_{0}^{c f} x, y\right) \varphi(x, y, \bar{z})}[\bar{a}], c \in N^{1}, c \in \operatorname{Dom}\left(\leq_{N_{1}, \bar{a}}^{\varphi}\right)$ and in $N^{1}$ the element $c$ is $\leq_{N^{1}, \bar{a}^{-}}^{\varphi}$ above all $\mathrm{d} \in \operatorname{Dom}\left(\leq_{M^{1}, \overline{\mathrm{a}}}^{\varphi}\right)$, then in $\mathrm{N}^{2}$ the element $\mathrm{c}$ is $\leq_{\mathrm{N}^{2}, \overline{\mathrm{a}}^{-}}^{\varphi}$-above all $\mathrm{d} \in \operatorname{Dom}\left(\leq_{\mathrm{M}^{2}, \overline{\mathrm{a}}}^{\varphi}\right)$.

3) For $\bar{M}^{1}, \bar{M}^{2} \in K_{\alpha}$ let $\bar{M}^{1} \leq \bar{M}^{2}$ means $\gamma<\beta<\alpha \Rightarrow\left(M_{\gamma}^{1}, M_{\beta}^{1}\right) \leq\left(M_{\gamma}^{2}, M_{\beta}^{2}\right)$; similarly $\bar{M}^{1} \leq_{\kappa_{\alpha}} \bar{M}^{2}$ means $\bar{M}^{1}, \bar{M}^{2} \in K_{\alpha}$ and $\gamma<\beta<\alpha \Rightarrow\left(M_{\delta}^{1}, M_{\beta}^{1}\right) \leq_{k}\left(M_{\gamma}^{2}, M_{\beta}^{2}\right)$.

4) For $\bar{M} \in \mathrm{K}_{\alpha}$, $\mathrm{D}$ an ultrafilter on $\lambda$ we define $\overline{\mathrm{N}}=\bar{M}^{\lambda} / \mathrm{D}, \mathbf{j}_{\mathrm{M}, \mathrm{D}}=\mathbf{j}_{\bar{M}, \mathrm{D}}^{\lambda}$ naturally: $\mathrm{N}_{\beta}=\mathrm{M}_{\beta}^{\lambda} / \mathrm{D}$ for $\beta<\alpha$ and $\mathbf{j}_{\bar{M}, \mathrm{D}}=\cup\left\{\mathbf{j}_{\mathrm{M}_{\beta}, \mathrm{D}}: \beta<\alpha\right\}$, recalling 3 , 
Fact 13. 0) For $\bar{M}^{1}, \bar{M}^{2} \in \mathrm{K}_{\alpha}$ we have

(a) $\bar{M}^{1} \leq_{\mathrm{K}_{\alpha}} \bar{M}^{2}$ iff $\mathbf{m}\left(M^{1}\right) \subseteq \mathbf{m}\left(\bar{M}^{2}\right)$

(b) $\left(\mathbf{m}\left(\bar{M}^{\ell}\right)\left\lceil P^{M_{\beta}}\right)\left\lceil\tau_{\top}=M_{\beta}^{\ell}\right.\right.$

(c) $\bar{M}^{1} \leq_{\mathrm{K}_{\alpha}} \bar{M}^{1}$ implies $\bar{M}^{1} \leq \bar{M}^{2}$.

1) $\left(\mathrm{K}_{\alpha}, \leq\right)$ and $\left(\mathrm{K}_{\alpha}, \mathrm{K}_{\alpha}\right)$ are partial orders.

2a) If $\bar{M}^{1} \leq_{K_{\alpha}} \bar{M}^{2}$ in $K_{\alpha}$ and $0<\gamma<\beta \leq \alpha$ then $\left(\bigcup_{\varepsilon<\gamma} M_{\varepsilon}^{1}, \bigcup_{\varepsilon<\beta} M_{\varepsilon}^{1}\right) \leq\left(\bigcup_{\varepsilon<\gamma} M_{\varepsilon}^{2}, \bigcup_{\varepsilon<\beta} M_{\varepsilon}^{2}\right)$ moreover $\left\langle\bigcup_{i<1+\varepsilon} M_{i}^{1}: 1+\varepsilon \leq \alpha\right\rangle \leq K_{\varepsilon}\left\langle\bigcup_{i<1+\varepsilon} M_{i}^{2}: 1+\varepsilon \leq \alpha\right\rangle$ where $\xi$ is $\alpha$ if $\alpha<\omega$ and is $\alpha+1$ if $\alpha \geq \omega$.

2b) If $\left\langle\bar{M}^{i}: i<\delta\right\rangle$ is a $\leq_{K_{\alpha}}$-increasing sequence (of members of $K_{\alpha}$ ) and we define $\bar{M}^{\delta}=\left\langle M_{\varepsilon}^{\delta}: \varepsilon<\right.$ $\alpha\rangle$ by $M_{\varepsilon}^{\delta}=\cup\left\{M_{\varepsilon}^{i}: i<\delta\right\}$ then $i<\delta \Rightarrow \bar{M}^{i} \leq K_{\alpha} \bar{M}^{\delta}$ and the sequence $\left\langle\bar{M}^{i}: i \leq \delta\right\rangle$ is continuous in $\delta$.

3) In part (2b), if in addition $i<\delta \Rightarrow \bar{M}^{i} \leq_{K_{\alpha}} \bar{N}$ so $\bar{N} \in K_{\alpha}$ then $\bar{M}^{\delta} \leq_{K_{\alpha}} \bar{N}$.

4) In part (2b), if $\delta<\mu^{+}$and $i<\delta \Rightarrow \bar{M}^{i} \in \mathrm{K}_{\mu}^{\alpha}$ then $\bar{M}^{\delta} \in \mathrm{K}_{\mu}^{\alpha}$.

5) If $\bar{M} \leq K_{\alpha} \bar{N}$ and $Y_{\varepsilon} \subseteq N_{\varepsilon}$ for $\varepsilon<\alpha$ and $\Sigma\left\{\left\|M_{\varepsilon}\right\|+\left|Y_{\varepsilon}\right|: \varepsilon<\alpha\right\}+|\tau|+|\alpha| \leq \lambda$ then there is $\bar{N}^{\prime} \in \mathrm{K}_{\lambda}^{\alpha}$ such that $\overline{\mathrm{M}} \leq_{\mathrm{K}_{\alpha}} \overline{\mathrm{N}}^{\prime} \leq_{\mathrm{K}_{\alpha}} \overline{\mathrm{N}}$ and $\varepsilon<\alpha \Rightarrow \mathrm{Y}_{\varepsilon} \subseteq \mathrm{N}_{\varepsilon}^{\prime}$.

6) Assume $\bar{M}^{i} \in K_{\mu}^{\alpha(i)}$ for $i<\delta<\mu^{+},\langle\alpha(i): i<\delta\rangle$ is a non-decreasing sequence of ordinals and $i<j<\delta \Rightarrow \bar{M}^{i} \leq_{K_{\alpha(i)}}, \bar{M}^{j}\left\lceil\alpha(i)\right.$ and we define $\alpha(\delta)=\cup\{\alpha(i): i<\delta\}, \bar{M}^{\delta}=\left\langle M_{\beta}^{\delta}: \beta<\alpha(\delta)\right\rangle$ where $M_{\beta}^{\delta}=\cup\left\{M_{\beta}^{i}: \beta<\delta\right.$ satisfies $\left.\beta<\alpha(i)\right\}$ then $\bar{M}^{\delta} \in K_{\mu}^{\alpha(\delta)}$ and $i<\delta \Rightarrow \bar{M}^{i} \leq_{K_{\alpha(i)}} \bar{M}^{\delta}\lceil\alpha(i)$.

7) If $\bar{M}^{\ell} \leq_{K_{\alpha}} \bar{N}$ for $\ell=1,2$ and $\left[a \in \mathbf{m}\left(\bar{M}^{1}\right) \Rightarrow a \in \mathbf{m}\left(\bar{M}^{2}\right)\right]$ then $\bar{M}^{1} \leq_{K_{\alpha}} \bar{M}^{2}$.

8) Parts (2)-(7) holds also when we replace $\leq_{K_{\alpha}}$ by $\leq$.

Demostración. Check.

Fact 14. 1) If $\left(M_{0}, M_{1}\right) \in K_{\mu}^{2}$ and $\left(M_{0}, M_{1}^{\prime}\right) \in K_{\mu}^{2}$ then there are $M_{2}$, f such that

(a) $M_{1}^{\prime} \prec M_{2} \in K_{\mu}$

(b) $f$ is an elementary embedding of $M_{1}$ into $M_{2}$

(c) $f \uparrow M_{0}=\operatorname{id}_{M_{0}}$

(d) $\left(M_{0}, M_{1}^{\prime}\right) \leq_{K_{2}}\left(f\left(M_{1}\right), M_{2}\right)$. 
2) If $\bar{M} \in \mathrm{K}_{\alpha}, \bar{x}=\left\langle\chi_{\varepsilon}: \varepsilon<\zeta\right\rangle$ and $\Gamma$ is a set of first order formulas from $\mathbb{L}\left(\tau_{\alpha}^{+}\right)$in the variables $\bar{x}$ with parameters from the model $\mathbf{m}(\bar{M})$ finitely satisfiable in $\mathbf{m}(M)$ such that $\varepsilon<\zeta \Rightarrow \underset{\beta<\alpha}{\bigvee} P_{\beta}\left(x_{\varepsilon}\right) \in \Gamma$, then there is $\bar{N} \in \mathrm{K}_{\alpha}$ such that $\bar{M} \leq_{\kappa_{\alpha}} \bar{N}$ and $\Gamma$ is realized in $\mathbf{m}(\overline{\mathrm{N}})$.

3) If $\Gamma$ is a type over $\mathbf{m}_{0}(\bar{M})$ of cardinality $3<\operatorname{cf}(\alpha) \underline{\text { then }}$ it is included in some $\Gamma^{\prime}$ as in part (2).

4) If $\bar{M} \in K_{\mu}^{\alpha}, D$ an ultrafilter on $\theta$ and $M_{\beta}^{\prime}=\left(M_{\beta}\right)^{\theta} / D$ for $\beta<\alpha$ then

(a) $\bar{M}^{\prime}=\left\langle M_{\beta}^{\prime}: \beta<\alpha\right\rangle \in K_{\alpha}$

(b) $\mathbf{j}_{\bar{M}, \mathrm{D}}^{\ominus}:=\cup\left\{\mathbf{j}_{\mathrm{M}_{\beta}, \mathrm{D}}^{\ominus}: \beta<\alpha\right\}$ is a $\leq_{\mathrm{K}_{\alpha}}$-embedding of $\bar{M}$ into $\bar{M}^{\prime}$, i.e.

$(\mathrm{b})^{\prime}\left\langle\mathbf{j}_{\mathrm{M}_{\beta}, \mathrm{D}}^{\theta}\left(\mathrm{M}_{\beta}\right): \beta<\alpha\right\rangle=\bar{M}^{\prime} \leq_{\mathrm{K}_{\alpha}}\left\langle\mathrm{M}_{\beta}^{\prime}: \beta<\alpha\right\rangle$, so

(c) for many $Y \in\left[\cup\left\{M_{\beta}^{\prime}: \beta<\alpha\right\}\right]^{\mu}$ we have $\mathbf{j}_{\bar{M}, D}^{\theta}(\bar{M}) \leq K_{\alpha}\left\langle M_{\beta}^{\prime} \uparrow Y: \beta<\alpha\right\rangle \in K_{\mu}^{\alpha}$; see $[13(5)$, 17(3).

Demostración. 1) See [Sh:199, §4]; just let $D$ be a regular ultrafilter on $\lambda \geq\left\|M_{1}\right\|+|\tau|$, let $g$ an elementary embedding of $M_{1}$ into $\left(M_{0}\right)^{\lambda} / D$ extending $\mathbf{j}=\mathbf{j}_{M_{0}, D}^{\lambda}$, necessarily exists.

Lastly, let $M_{2} \prec\left(M_{1}^{\prime}\right)^{\lambda} / D$ include $\mathbf{j}_{M_{1}, D}^{\lambda}\left(M_{1}^{\prime}\right) \cup g\left(M_{1}\right)$ be of cardinality $\mu$. Identifying $M_{1}^{\prime}$ with $\mathbf{j}_{M_{1}^{\prime}, \mathrm{D}}^{\lambda}\left(M_{1}^{\prime}\right) \prec\left(M_{1}^{\prime}\right)^{\lambda} / D$ we are done.

2) Similarly.

3) Trivial.

4) Should be clear.

Definition 15. $K_{\alpha}^{\mathrm{ec}}$ is the class of $\bar{M} \in \mathrm{K}_{\alpha}$ such that: if $\bar{M} \leq_{K_{\alpha}} \overline{\mathrm{N}} \in \mathrm{K}_{\alpha}$, then $\mathbf{m}(\overline{\mathrm{M}}) \leq_{\Sigma_{1}} \mathbf{m}(\overline{\mathrm{N}})$, i.e. $(*)$ below and $K_{\lambda}^{\text {ec, } \alpha}=K_{\alpha}^{\text {ec }} \cap K_{\lambda}$ where

(*) if $a_{1}, \ldots, a_{n} \in \mathbf{m}(\bar{M}), b_{1}, \ldots, b_{k} \in \mathbf{m}(\bar{N}), \varphi \in \mathbb{L}\left(\tau_{\alpha}^{+}\right)$is quantifier free and $\mathbf{m}(\bar{N}) \models$

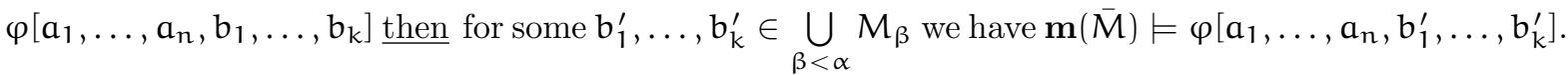

Claim 16. 1) $\mathrm{K}_{\mu}^{\mathrm{ec}, \alpha}$ is dense in $\mathrm{K}_{\mu}^{\alpha}$ when $\mu \geq\left|\tau_{\mathrm{T}}\right|+|\alpha|$ of course.

2) $\mathrm{K}_{\mu}^{\mathrm{ec}, \alpha}$ is closed under union of increasing chains of length $<\mu^{+}$.

3) In Definition 15, if $|\alpha|+\left|\tau_{\mathrm{T}}\right| \leq \mu$ and $\bar{M} \in \mathrm{K}_{\mu}^{\alpha}$ then without loss of generality $\overline{\mathrm{N}} \in \mathrm{K}_{\mu}^{\alpha}$.

Demostración. 1) Given $\bar{M}_{0} \in K_{\mu}^{\alpha}$ we try to choose $\bar{M}_{\varepsilon} \in K_{\mu}^{\alpha}$ by induction on $\varepsilon<\mu^{+}$such that $\left\langle\bar{M}_{\zeta}: \zeta \leq \varepsilon\right\rangle$ is $\leq_{\kappa_{\alpha}}$-increasing continuous and $\varepsilon=\zeta+1 \Rightarrow \mathbf{m}\left(\bar{M}_{\zeta}\right) \not \Sigma_{1} \mathbf{m}\left(M_{\varepsilon}\right)$. For $\varepsilon=0$ the sequence is given, for $\varepsilon$ limit use 13(2), for $\varepsilon=\zeta+1$ if we cannot choose then by 13(5) we get

\footnotetext{
3 also if $\operatorname{cf}(\alpha)=1$, i.e. $\alpha$ is a successor ordinal
} 
$\bar{M}_{\zeta} \in \mathrm{K}_{\mu}^{\mathrm{ec}, \alpha}$ is as required. But if we succeed to choose $\left\langle\bar{M}_{\varepsilon}: \varepsilon<\mu^{+}\right\rangle$we get contradiction by Fodor lemma.

2) Think on the definitions.

3) By LST.

Claim 17. 1) If $\bar{M}, \bar{N} \in \mathrm{K}_{\mu}^{\alpha}$ and $\bar{M} \leq_{\Sigma_{1}} \bar{N}$ and $\bar{N} \in \mathrm{K}_{\alpha}^{\mathrm{ec}} \underline{\text { then }} \overline{\mathrm{M}} \in \mathrm{K}_{\alpha}^{\mathrm{ec}}$.

2) If $\overline{\mathrm{N}} \in \mathrm{K}_{\mu}^{\mathrm{ec}, \alpha}, \mathrm{Y} \subseteq \mathbf{m}_{0}(\overline{\mathrm{N}})$ and $\lambda=\left|\tau_{\mathrm{T}}\right|+|\alpha|+|\mathrm{Y}| \underline{\text { then }}$ there is $\bar{M} \in \mathrm{K}_{\lambda}^{\mathrm{ec}, \alpha}$ such that $\bar{M} \leq_{\mathrm{K}_{\alpha}} \overline{\mathrm{N}}$ and $\mathrm{Y} \subseteq \mathbf{m}_{0}(\bar{M})$.

3) Assume $\bar{M}^{\ell} \in \mathrm{K}_{\mu}^{\alpha}$ and $\bar{M}^{0} \leq_{\mathrm{K}_{\alpha}} \bar{M}^{1}$ and $\bar{M}^{0} \leq \bar{M}^{2}$. If $\bar{M}^{0} \in \mathrm{K}_{\mu}^{\mathrm{ec}, \alpha}, \bar{M}^{0} \leq_{\mathrm{K}_{\alpha}} \bar{M}^{2}$ or $\mathbf{m}\left(\bar{M}^{0}\right) \leq \Sigma_{1}$ $\mathbf{m}\left(\bar{M}^{2}\right)$, then we can find $\left(\overline{\mathrm{N}}, \mathrm{f}_{2}\right)$ such that:

$\bar{M}^{1} \leq_{K_{\alpha}} \bar{N} \in K_{\mu}^{\alpha}$, moreover $\bar{N} \in K_{\mu}^{e c, \alpha}$ and $f_{2}$ is a $\leq_{K_{\alpha}}$-embedding of $\bar{M}^{2}$ into $\bar{N}$ over $\bar{M}^{0}$.

Demostración. 1) By part (3).

2) By part (1) and the LST argument.

3) By the definition of $\bar{M}^{0} \in \mathrm{K}_{\mu}^{\mathrm{ec}, \alpha}$ in both cases we can assume $\bar{M}^{0} \leq_{\Sigma_{1}} \bar{M}^{2}$. Let $\overline{\mathbf{a}}=\left\langle\mathrm{a}_{\varepsilon}: \varepsilon<\zeta\right\rangle$ list the elements of $\mathbf{m}\left(\bar{M}^{2}\right)$ and let $\Gamma=\operatorname{tp}_{\mathrm{qf}}\left(\overline{\mathbf{a}}, \emptyset, \mathbf{m}\left(\bar{M}^{2}\right)\right)=\left\{\varphi\left(x_{\varepsilon_{0}}, \ldots, x_{\varepsilon_{n-1}}, \bar{b}\right): \varphi \in \mathbb{L}\left(\tau_{\alpha}^{+}\right)\right.$is quantifier free, $\overline{\mathrm{b}} \subseteq \mathbf{m}\left(\bar{M}^{0}\right)$ and $\left.\mathbf{m}\left(\bar{M}^{2}\right) \models \varphi\left[\mathrm{a}_{\varepsilon_{0}}, \ldots, \mathrm{a}_{\varepsilon_{n-1}}, \overline{\mathrm{b}}\right]\right\}$; note that $\left.\mathrm{P}_{\beta}\left(\mathrm{x}_{\varepsilon}\right)\right)^{\mathbf{t}(\varepsilon, \beta)} \in \Gamma$ when $\beta<\alpha, \varepsilon<\zeta$ and $\mathbf{t}(\varepsilon, \beta)$ is the truth value of $a_{\varepsilon} \in M_{\beta}^{2}$.

Now let $\mathrm{D}$ be a regular ultrafilter on $\lambda=\left\|\mathbf{m}\left(\bar{M}^{2}\right)\right\|$ and use [14 $(2),(3)$. This is fine to get $\left(f_{2}, \bar{N}\right)$ with $\bar{N} \in K_{\alpha}$ and by 13 (5) without loss of generality $\bar{N} \in K_{\mu}^{\alpha}$ and by 16(1) without loss of generality $\bar{N} \in K_{\mu}^{\mathrm{ec}, \alpha}$.

Claim 18. 1) $\left(\mathrm{K}_{\mu}^{\mathrm{ec}, \alpha}, \leq_{\mathrm{K}_{\mu}^{\alpha}}\right)$ has the JEP.

2) Suppose $\bar{M}^{1}, \bar{M}^{2} \in K_{\mu}^{\alpha}, \beta \leq \alpha$, f is an elementary embedding of $\bigcup_{\gamma<\beta} M_{\gamma}^{1}$ into $\bigcup_{\gamma<\beta} M_{\gamma}^{2}$ such that $\left\langle f\left(M_{\gamma}\right): \gamma<\beta\right\rangle \leq_{K_{\mu}}\left\langle M_{\gamma}^{2}: \gamma<\beta\right\rangle$, equivalently $f$ is an embedding of $\mathbf{m}\left(\bar{M}^{1} \uparrow \beta\right)$ into $\mathbf{m}\left(\bar{M}^{2} \uparrow \beta\right)$ (so if $\beta=0$ then $f=\emptyset$ and there is no demand).

Then we can find $\bar{M}^{3}, f^{+}$such that:

(a) $\bar{M}^{2} \leq_{K_{\mu}} \bar{M}^{3} \in K_{\mu}^{\alpha}$

(b) $f \subseteq f^{+}$

(c) $\mathrm{f}^{+}$is an elementary embedding of $\bigcup_{\gamma<\alpha} M_{\gamma}^{1}$ into $\underset{\gamma<\alpha}{\bigcup} M_{\gamma}^{3}$

(d) $\left.\left\langle f^{+}\left(M_{\gamma}^{1}\right)\right): \gamma<\alpha\right\rangle \leq_{K_{\alpha}}\left\langle M_{\gamma}^{3}: \gamma<\alpha\right\rangle$.

Demostración. 1) A special case of part (2) recalling 16(1). 
2) By induction on $\alpha$.

$\alpha=0$ : nothing to do.

$\underline{\beta=\alpha}$ : nothing to do.

$\underline{\alpha=1}$ : so $\beta=0$ which is trivial or $\beta=\alpha$, a case done above.

$\underline{\alpha \text { successor: }}$ by the induction hypothesis and transitive nature of conclusion replacing $\bar{M}^{2}$ without loss of generality $\beta=\alpha-1$, then use 14(1).

$\underline{\alpha \text { limit: }}$ By $\alpha-\beta$ successive uses of induction hypothesis using [13(2b).

Conclusion 19. ( $\left.\mathrm{K}_{\alpha}^{\mathrm{ec}}, \leq_{\mathrm{K}_{\alpha}}\right)$, or formally $\mathfrak{k}=\left(\mathrm{K}_{\mathfrak{k}}, \leq_{\mathfrak{k}}\right)$ defined by $\mathrm{K}_{\mathfrak{k}}:=\left\{\mathbf{m}(\bar{M}): \bar{M} \in \mathrm{K}_{\alpha}^{\text {ec }}\right\}, \mathbf{m}\left(\bar{M}^{1}\right) \leq_{\mathfrak{k}}$ $\mathbf{m}\left(\bar{M}^{2}\right) \Leftrightarrow \mathbf{m}\left(M^{1}\right) \subseteq \mathbf{m}\left(\bar{M}^{2}\right)$, is an a.e.c. with amalgamation, the JEP and $\operatorname{LST}(\mathfrak{k}) \leq\left|\tau_{\mathrm{T}}\right|+|\alpha|+\boldsymbol{\Upsilon}_{0}$.

Demostración. By the above, on a.e.c. see [Sh:h, Ch.I], i.e. [Sh:88r] and history there.

Fact 20. Assume $\lambda=\lambda^{<\lambda}>\left|\tau_{\mathrm{T}}\right|+\boldsymbol{\aleph}_{0}+|\alpha|$. Then there is $\bar{M}$ such that

(a) $\bar{M} \in \mathrm{K}_{\alpha}^{\mathrm{ec}}$ is universal for $\left(\mathrm{K}_{\alpha}^{\mathrm{ec}}, \mathrm{K}_{\alpha}\right)$ in cardinality $\lambda$

(b) $\mathbf{m}(\bar{M})$ is model homogeneous for $\left(\mathrm{K}_{\alpha}^{\mathrm{ec}}, \leq_{\mathrm{K}_{\alpha}}\right)$ of cardinality $\lambda$

(c) $\mathbf{m}(\bar{M})$ is sequence $\left(\Sigma_{1}\left(\tau_{\alpha}^{+}\right), \lambda\right)$-homogeneous, see $4(3)$.

Demostración. Clause $(\mathrm{a})+(\mathrm{b})$ are straight by $17+$ 18 $(1)$, or use 19 and see [Sh:h, Ch.I,, 2$]=$ Sh:88r, §2]. Now clause (c) follows: just think.

Fact 21. Assume $\bar{M} \in \mathrm{K}_{\mu}^{\alpha}, \beta+1<\alpha, \ell \in\{0,1\}$ and $\mathrm{M}_{\beta} \models \mathrm{P}_{\left(\mathrm{Q}_{\ell}^{\mathrm{cf}} x, y\right) \varphi(x, y, \bar{z})}[\overline{\mathrm{a}}]$ then there are $\overline{\mathrm{N}}, \mathrm{c}$ such that $\bar{M} \leq_{K_{\alpha}} \bar{N} \in K_{\mu}^{\alpha}$ and:

$(*)_{1}$ if $\ell=1$ then $c \in \operatorname{Dom}\left(\leq_{\mathbb{N}_{\beta}, \bar{a}}\right)$ and $c$ is $\leq_{\mathbb{N}_{\gamma}, \bar{a}}$-above $\mathrm{d} \in \operatorname{Dom}\left(\leq_{\mathcal{M}_{\gamma}, \overline{\mathrm{a}}}\right)$ for any $\gamma \in[\beta, \alpha)$

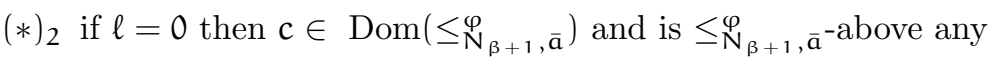

$$
\mathrm{d} \in \operatorname{Dom}\left(\leq_{\mathrm{N}_{\beta}, \overline{\mathrm{a}}}\right)
$$

Demostración. First assume $\ell=1$, without loss of generality $\beta=0$ as we can let $\bar{N} \uparrow \beta=\bar{M} \uparrow \beta$.

By $13(2 \mathrm{a})$ wlog $\bar{M}$ is increasing continuous; we prove by induction on $\alpha$ so easily without loss of generality $\alpha=2$. Now this is obvious by Sh:43, Sh:199]; in details by Sh:43] there is a $\mu^{+}$ saturated model $M_{*}$ of $T$ such that $M_{1} \prec M_{*}$ and $M_{*} \models C_{*} T^{*}$ whenever, e.g. $\mu^{++} \in C_{*} \wedge \mu^{+} \notin C_{*}$. Let $\left\{\varphi_{i}\left(x, y, \bar{a}_{i}^{*}\right): i<\mu\right\}$ list $\left\{\varphi\left(x, y, \bar{a}^{\prime}\right): \varphi \in \mathbb{L}\left(\tau_{T}\right), M_{0} \models P_{\left(Q_{0}^{\text {cfx } x y}\right) \varphi(x, y ; z)}\left[\bar{a}^{\prime}\right]\right\}$, and for each $i<\mu$ let $\left\langle c_{i, \varepsilon}: \varepsilon<\mu^{+}\right\rangle$be $\leq_{M_{*}, \bar{a}_{i}^{*}}^{\varphi_{i}}$-increasing and cofinal. For $\varepsilon<\mu^{+}$let $f_{\varepsilon}$ be an elementary embedding of $M_{1}$ into $M_{*}$ over $M_{0}$ such that: 
(*) if $\mathrm{c} \in \operatorname{Dom}\left(\leq_{M_{*}, \bar{a}_{i}^{*}}^{\varphi_{i}}\right)$ is a $\leq_{M_{*}, \bar{a}_{i}^{*}}^{\varphi_{i}}$ upper bound of $\operatorname{Dom}\left(\leq_{M_{0}, \bar{a}_{i}^{*}}^{\varphi_{i}}\right)$, then $c_{i, \varepsilon} \leq_{M_{*}, \bar{a}_{i}^{*}}^{\varphi_{i}}$ c.

Let $c_{*} \in M_{*}$ be a $\leq_{M_{*}}^{\varphi}, \bar{a}$-upper bound of $\operatorname{Dom}\left(\leq_{M_{0}, \bar{a}}^{\varphi}\right)$. Choose $N_{0} \prec M_{*}$ of cardinality $\mu$ be such that $M_{0} \cup\left\{c_{*}\right\} \subseteq N_{0}$ and choose $\varepsilon<\mu^{+}$large enough such that:

(*) if $i<\mu$ and $d \in N_{O}$ is a $\leq_{M_{*}, \bar{a}_{i}}^{\varphi_{i}}$-upper bound of $\operatorname{Dom}\left(\leq_{M_{i}, \bar{a}_{i}}^{\varphi_{i}}\right)$ then $d \leq_{M_{*}, \bar{a}_{i}}^{\varphi_{i}} c_{i, \varepsilon}$.

Let $N_{1} \prec M_{*}$ be of cardinality $\mu$ be such that $N_{0} \cup f_{\varepsilon}\left(M_{1}\right) \subseteq N_{1}$. Renaming, $f_{\varepsilon}$ is the identity and $\left(\mathrm{N}_{0}, \mathrm{~N}_{1}\right)$ is as required.

Second, assume $\ell=0$ is even easier (again without loss of generality first, $\alpha=\beta+2$ and second $\beta=0, \alpha=2$ and use $N_{0}=M_{0}, N_{1}$ satisfies $M_{1} \prec N_{1}$ and $\left\|N_{1}\right\|=\mu$ and $N_{1}$ realizes the relevant upper).

Conclusion 22. In 20 the model $M^{*}=\mathbf{m}\left(\bar{M}^{*}\right)=\bigcup_{\beta<\alpha} M_{\beta}^{*}$ satisfies

(a) if $M^{*} \models P_{\left(Q_{1}^{\text {cf }} x, y\right) \varphi}[\overline{\mathrm{a}}]$ then the order $\leq_{M^{*}, \overline{\mathrm{a}}}^{\varphi}$ has cofinality $\lambda$

(b) if $\alpha$ is a limit ordinal and $M^{*} \models P_{\left(Q_{0}^{\text {cf }} x, y\right) \varphi}[\bar{a}]$ then the linear order $\leq_{M^{*}, \bar{a}}^{\varphi}$ has cofinality $\operatorname{cf}(\alpha)$

(c) $M^{*}$ is $\operatorname{cf}(\alpha)$-saturated

(d) if $\lambda \in C$ and $\operatorname{cf}(\alpha) \in \operatorname{Reg} \backslash C$ then $M^{*}$ is a model of $T^{*}$.

Claim 23. Assume $\bar{M} \in \mathrm{K}_{\alpha}^{\mathrm{ec}}$. If $\zeta \leq \mu$ and $\overline{\mathrm{a}}, \overline{\mathrm{b}} \in \zeta\left(\mathrm{M}_{0}^{*}\right)$ realize the same type (equivalently q.f. type) in $M_{0}$ then they realize the same $\Sigma_{1}$-type in $\mathbf{m}(\bar{M})$.

Demostración. We choose $\left(\mathrm{N}_{\beta}, \mathrm{f}_{\beta}, \mathrm{g}_{\beta}, \mathrm{h}_{\beta}\right)$ by induction on $\beta<\alpha$ such that:

(a) $\mathrm{N}_{\beta}$ is a model of $\mathrm{T}$

(b) $N_{\beta}$ is $\prec$-increasing continuous with $\beta$

(c) $f_{\beta}, g_{\beta}$ are $\leq_{K_{1+\beta}}$-embedding of $\bar{M} \uparrow(1+\beta)$ into $\left\langle N_{\gamma}: \gamma<1+\beta\right\rangle \in K_{1+\beta}$

(d) $f_{0}(\bar{a})=g_{0}(\bar{b})$

(e) if $\gamma<\beta$ then $\mathrm{f}_{\gamma} \subseteq \mathrm{f}_{\beta}, \mathrm{g}_{\gamma} \subseteq \mathrm{g}_{\beta}$. 
For $\beta=0$ this speaks just on $\operatorname{Mod}_{\mathrm{T}}$.

For $\beta$ successor use 14.

For $\beta$ limit as in the successor case, recalling we translated it to the successor case (by 13(2a)).

Having carried the induction $f=\cup\left\{f_{\beta}: \beta<\alpha\right\}$ and $g=\cup\left\{g_{\beta}: \beta<\alpha\right\}$ are $\leq_{K_{\alpha}}$-embedding of $\bar{M}$ into $\bar{N}=\left\langle\mathrm{N}_{\beta}: \beta<\alpha\right\rangle$. By $16(1)$ there is $\overline{\mathrm{N}}^{\prime} \in \mathrm{K}_{\alpha}^{\mathrm{ec}}$ which is $\leq_{\mathrm{K}_{\alpha}}$-above $\overline{\mathrm{N}}$. Now as $\overline{\mathrm{M}} \in \mathrm{K}_{\alpha}^{\mathrm{ec}}$, the $\Sigma_{1}$-type of $\bar{a}$ in $\mathbf{m}(\bar{M})$ is equal to the $\Sigma_{1}$-type of $f(\bar{a})$ in $\mathbf{m}\left(\bar{N}^{\prime}\right)$, and the $\Sigma_{1}$-type of $\bar{b}$ in $\mathbf{m}(\bar{M})$ is equal to the $\Sigma_{1}$-type of $f(\bar{a})$ in $\mathbf{m}\left(\bar{N}^{\prime}\right)$. But $f(\bar{a})=f_{0}(\bar{a})=g_{0}(\bar{b})=g(\bar{b})$, so we have gotten the promised equality of $\Sigma_{1}$-types.

Observation 24. 1) If $\bar{M} \in K_{\alpha}^{\text {ec }}$ and $\beta<\alpha$ then $\bar{M}^{\prime}: \bar{M}\left\lceil[\beta, \alpha)=\left\langle M_{\beta+\gamma}: \gamma<\alpha-\beta\right\rangle\right.$ belongs to $\mathrm{K}_{\alpha-\beta}^{\mathrm{ec}}$.

2) If $\bar{M} \in K_{\alpha}, \beta<\alpha$ and $\bar{M} \uparrow[\beta, \alpha) \leq_{\kappa_{\alpha, \beta}} \bar{N}^{\prime}$ then for some $\bar{N} \in K_{\alpha}$ we have $\bar{M} \leq_{\kappa_{\alpha}} \bar{N}$ and $\overline{\mathrm{N}} \uparrow[\beta, \alpha)=\overline{\mathrm{N}}^{\prime}$.

Demostración. 1) If not, then there is $\bar{N}^{\prime} \in \mathrm{K}_{\alpha-\beta}$ such that $\bar{M}^{\prime} \leq_{K_{\alpha-\beta}} \bar{N}^{\prime}$ but $\mathbf{m}\left(\bar{M}^{\prime}\right) \not \Sigma_{\Sigma_{1}} \mathbf{m}\left(\bar{N}^{\prime}\right)$. Define $\bar{N}=\left\langle N_{\gamma}: \gamma<\alpha\right\rangle$ by: $N_{\gamma}$ is $M_{\gamma}$ if $\gamma<\beta$ and is $N_{\gamma-\beta}^{\prime}$ if $\gamma \in[\beta, \alpha)$. Easily $\bar{M} \leq_{K_{\alpha}} \bar{N} \in K_{\alpha}$ but $\mathbf{m}(\bar{M}) \not \Sigma_{1} \mathbf{m}(\overline{\mathrm{N}})$, contradiction to the assumption $\bar{M} \in \mathrm{K}_{\alpha}^{\mathrm{ec}}$.

2) The proof is included in the proof of part (1).

Claim 25. In 20 for each $\beta<\alpha$ we have

(a) $\left\langle M_{\beta+\gamma}^{*}: \gamma<\alpha-\beta\right\rangle$ is homogeneous universal for $K_{\mu}^{\alpha-\beta}$

(b) if $\alpha=\alpha-\beta$, i.e. $\beta+\alpha=\alpha$ then there is an isomorphism from $\bar{M}^{*}$ onto $\left\langle M_{\beta+\gamma}^{*}: \gamma<\alpha-\beta\right\rangle$, in fact, we can determine $f(\bar{a})=\bar{b}$ if $\bar{a} \in \zeta\left(M_{0}^{*}\right), \bar{b} \in \zeta\left(M_{\beta}^{*}\right)$ and $\operatorname{tp}\left(\bar{a}, \emptyset, M_{\beta}^{*}\right)=\operatorname{tp}\left(\bar{b}, \emptyset, M_{\beta}^{*}\right)$.

Demostración. Chase arrows as usual recalling 24.

\section{Demostración. Proof of Theorem[10}

Without loss of generality there is $\sigma=\sigma^{\Theta} \geq \mu$ such that $2^{\sigma}=\sigma^{+}$(why? let $\sigma=\sigma^{\theta}>\mu$ be regular, work in $\mathbf{V}^{\operatorname{Levy}\left(\sigma^{+}, 2^{\sigma}\right)}$ and use absoluteness argument, or choose set $A$ of ordinals such that $\mathcal{P}(\mu) \in \mathbf{L}[A]$ hence $\mathrm{T} \mathrm{T}^{*} \in \mathbf{L}[\mathrm{A}]$ and regular $\theta$ large enough such that $\mathbf{L}[\mathrm{A}] \models " 2^{\sigma}=\sigma^{+}$, work in $\mathbf{L}[A]$ a little more; and for the desired conclusion (there is a model of cardinality $\mu$ such that ...) it makes no difference). Let $\alpha=\kappa$ and let $\bar{M}^{*} \in K_{\lambda}^{\text {ec, }, \alpha}$ be as in 20 for $\lambda:=\sigma^{+}$and let $M_{*}=\cup\left\{M_{\beta}^{*}: \beta<\alpha\right\}$.

Now

$(*)_{1} \mathrm{M}_{*}$ is a model of $\mathrm{T}^{*}$ by the $\left\{\mu^{+}\right\}$-interpretation. 
[Why? By 22]]

$(*)_{2} M_{*}$ is $\theta$-saturated.

[Why? Clearly $M_{\beta}^{*}$ is $\theta$-saturated for each $\beta<\theta$. As $\theta$ is regular and $\left\langle M_{\beta}^{*}: \beta<\theta\right\rangle$ is increasing with union $M_{*}$, also $M_{*}$ is $\theta$-saturated.]

$(*)_{3} \quad M_{*}$ is strongly $\boldsymbol{\aleph}_{0}$-saturated and even strongly $\theta$-saturated, see Definition 4(1).

[Why? Let $\zeta<\theta$ and $\bar{a}, \bar{b} \in \zeta\left(M_{*}\right)$ realize the same q.f.-type (equivalently the first order type) in $M_{*}$. As $\zeta<\theta$ for some $\beta<\theta$ we have $\bar{a}, \bar{b} \in \zeta\left(M_{\beta}\right)$. Now by 25 we know that $\left\langle M_{\beta+\gamma}^{*}: \gamma<\theta\right\rangle \cong$ $\left\langle M_{\gamma}^{*}: \gamma<\theta\right\rangle$, and by 23 the sequences $\bar{a}, \bar{b}$ realize the same $\Sigma_{1}$-type in $\mathbf{m}\left(\left\langle M_{\beta+\gamma}^{*}: \gamma<\theta\right\rangle\right)$ hence by clause (c) of 20 there is an automorphism $\pi$ of it mapping $\bar{a}$ to $\bar{b}$. So $\pi$ is also an automorphism of $M_{*}$ mapping $\bar{a}$ to $\bar{b}$ as required.]

Lastly, we have to go back to models of cardinality $\mu=\mu^{<\theta} \geq \lambda+\kappa+2^{|\mathrm{T}|}$, this is done by the LST argument recalling 22

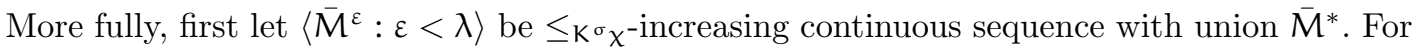
$\zeta<\theta$ and $\bar{a}, \bar{b} \in \zeta\left(M_{*}\right)$ let $f_{\bar{a}, \bar{b}}$ be an automorphism of $M_{*}$ mapping $\bar{a}$ to $\bar{b}$. Now the set of $\delta<\lambda$ satisfying $\circledast \delta$ below is a club of $\lambda$ hence if $\operatorname{cf}(\delta)=\chi$ then $M=\cup\left\{M_{\beta}^{\varepsilon}: \beta<\lambda\right\}$ is as required except of being of cardinality $\mu$, where

$\circledast_{\delta}(\mathrm{a}) \quad$ if $\varepsilon<\delta, \zeta<\theta$ and $\overline{\mathrm{a}}, \overline{\mathrm{b}} \in \zeta\left(\cup\left\{\mathrm{M}_{\beta}^{\varepsilon}: \beta<\alpha\right\}\right)$ realize the same $\Sigma_{1}$-type in $\bar{M}^{\zeta}$ then $\cup\left\{M_{\beta}^{\delta}: \beta<\alpha\right\}$ is closed under $f_{\bar{a}, \bar{b}}$ and under $f_{\bar{a}, \bar{b}}^{-1}$

(b) the witnesses for the cofinality work, i.e.

$\bullet_{1}$ if $\beta<\alpha, \bar{a} \in{ }^{\omega>}\left(M_{\beta}^{\delta}\right), M_{\beta}^{\delta} \models P_{\left(Q_{0}^{\text {cf }} y, z\right) \varphi(y, z, \bar{x})}[\bar{a}]$ then for some $\varepsilon<\delta$ we have $\bar{a} \subseteq M_{\beta}^{\varepsilon}$ and for every $\gamma \in(\beta, \alpha)$ there is $c=c_{\varphi, \bar{a}, \gamma} \in M_{\gamma+1}^{\varepsilon}$ which is a $\leq_{M_{\gamma+1}^{\varphi}, \bar{a}}$-upper bound of $\operatorname{Dom}\left(\leq_{M_{\gamma}^{\varepsilon}, \overline{\mathrm{a}}}^{\varphi}\right)$, hence this holds for any $\varepsilon^{\prime} \in[\varepsilon, \lambda)$

$\bullet_{2}$ if $\beta<\alpha, \bar{a} \in \omega>\left(M_{\beta}^{\gamma}\right)$ and $M_{\beta}^{\delta} \models P_{\left(Q_{1}^{\text {cf }} y, z\right) \varphi(y, z, \bar{x})}[\bar{a}]$ then for arbitrarily large $\varepsilon<\delta$ we have $\bar{a} \subseteq M_{\beta}^{\varepsilon}$ and there is $c=c_{\varphi, \bar{a}} \in M_{\beta}^{\varepsilon+1}$ which is a $\left(\leq_{M_{\gamma}^{\varepsilon+1}, \bar{a}}^{\varphi}\right)$-upper bound of $\operatorname{Dom}\left(\leq_{M_{\gamma}^{\varepsilon}, \overline{\mathbf{a}}}^{\varphi}\right)$ for every $\gamma \in[\beta, \alpha)$.

By a similar use of the LST argument we get a model of $\mathrm{T}^{*}$ of cardinal $\mu$.

Remark 26. If you do not like the use of (set theoretic absoluteness) you may do the following. Use 27 below, which is legitimate as 
(a) the class $\left(K_{\alpha}^{\text {ec }}, \leq_{\alpha}\right)$ is an a.e.c. with LST number $\leq|T|+\mathbf{K}_{0}$ and amalgamation, so 27(1) apply

(b) using $\Sigma_{1}$-types, it falls under [Sh:3] more exactly [Sh:54, so 27(3) apply

(c) we can define $\mathrm{K}_{\alpha}^{\mathrm{ec}(\varepsilon)}$ by induction on $\varepsilon \leq \omega$

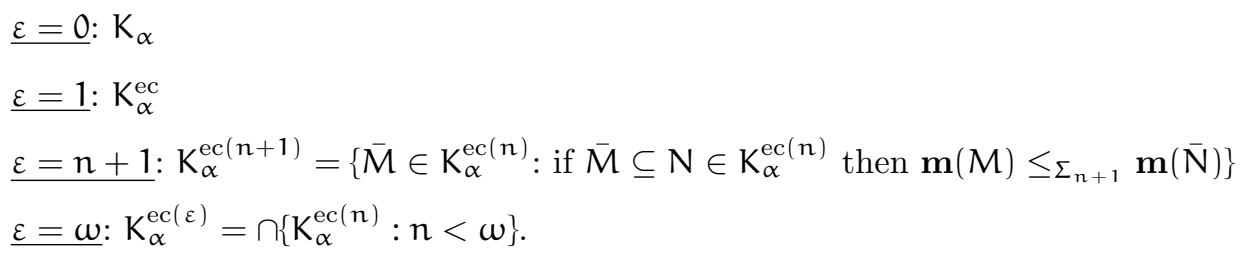

On $\mathrm{K}_{\alpha}^{\mathrm{ec}(\boldsymbol{\omega})}$ apply $27(2)$.

Remark 27. 1) Assume $\mathfrak{k}=\left(\mathrm{K}_{\mathfrak{k}}, \leq_{\mathfrak{k}}\right)$ is a a.e.c. satisfying amalgamation and the JEP with $\lambda>$ $\operatorname{LST}(\mathfrak{k})$ and $\mu=\mu^{<\lambda}$. For any $M \in K_{\mu}$ there is a strongly model $\lambda$-homogeneous $N \in K_{\mu}$ which $\leq_{\mathfrak{k}}$-extend $M$, which means: if $M \in K_{\mathfrak{k}}$ has cardinality $<\lambda$ and $f_{1}, f_{2}$ are $\leq_{\mathfrak{k}}$-embedding of $M$ into $\mathrm{N}$ then for some automorphism $g$ of $N$ we have $f_{2}=g \circ f_{1}$.

2) Let $\mathrm{D}$ be a good finite diagram as in [Sh:3 and let $K_{D}$ be as below in part (3) for $\Delta=\mathbb{L}(\tau)$. If $\lambda=\lambda^{<\theta} \geq|D|$ and $M \in K_{D}$ has cardinality $\lambda$ then there is $N \in K_{D}$ of cardinality $\lambda$ which $\prec$-extend $M$ and is strongly $(D, \theta)$-homogenous, i.e.

(a) if $\zeta<\theta, \bar{a}, \bar{b} \in \zeta N$ realizes the same type then some automorphism $f$ of $N$ maps $\bar{a}$ to $\bar{b}$

(b) $\mathrm{D}=\left\{\operatorname{tp}(\overline{\mathrm{a}}, \emptyset, \mathrm{N}): \overline{\mathrm{a}} \in{ }^{\omega>} \mathrm{N}\right\}$

3) Assume $\Delta \subseteq \mathbb{L}(\tau)$, not necessarily closed under negation, $\mathrm{D}$ is a set of $\Delta$-types, $\mathrm{K}_{\mathrm{D}}$ is the class of $\tau$-models such that $\bar{a} \in{ }^{\omega>} M \Rightarrow \operatorname{tp}_{\Delta}(\bar{a}, \emptyset, M) \in D$ and $M \leq_{D} N$ iff $M \subseteq N$ are from $K_{D}$ and $\bar{a} \in{ }^{\omega>} M \Rightarrow \operatorname{tp}_{\Delta}(\bar{a}, \emptyset, M)=\operatorname{tp}_{\Delta}(\bar{a}, \emptyset, N)$. Assume further $D$ is good, i.e. for every $M \in K_{D}$ and $\lambda$ there is a sequence $(D, \lambda)$-homogeneous model $N \in K_{D}$ which $\leq_{D}$-extends $M$. Then for every $\lambda=\lambda^{<\theta}>|\mathrm{T}|+\mathbf{X}_{0}$ and $M \in K_{D}$ of cardinality $\lambda$ there is a strongly sequence $(\Delta, \lambda)$-homogeneous.

Conclusion 28. 1) The logic $\mathbb{L}\left(Q_{C}^{\text {cf }}\right)$ has the strong $\boldsymbol{N}_{0}$-saturated model existence property (hence the strong $\mathbf{N}_{0}$-homogeneous model existence property).

2) If $\kappa=\operatorname{cf}(\kappa) \leq \operatorname{Min}(C)$ and $\kappa \leq \operatorname{Min}(\operatorname{Reg} \backslash C)$ then in part (1) we can replace $\boldsymbol{N}_{0}$ by $\kappa$.

Demostración. Choose $\chi \in \mathrm{C}, \mathrm{k} \in \mathrm{Reg} \backslash \mathrm{C}$ and apply 10. 


\section{Referencias}

[Mak85] Johann A. Makowsky, Compactnes, embeddings and definability, Model-Theoretic Logics (J. Barwise and S. Feferman, eds.), Springer-Verlag, 1985, pp. 645-716.

[Sh:h] Saharon Shelah, Classification Theory for Abstract Elementary Classes, Studies in Logic: Mathematical logic and foundations, vol. 18, College Publications, 2009.

[Sh:3] Finite diagrams stable in power, Annals of Mathematical Logic 2 (1970), 69-118.

[Sh:43] Generalized quantifiers and compact logic, Transactions of the American Mathematical Society 204 (1975), 342-364.

[Sh:54] The lazy model-theoretician's guide to stability, Logique et Analyse 18 (1975), 241308.

[Sh:88r] Abstract elementary classes near $\mathbf{\aleph}_{1}$, Chapter I Studies in Logic, College Publ. 18 (2009). 0705.4137. 0705.4137.

[MkSh:166] Alan H. Mekler and Saharon Shelah, Stationary logic and its friends. I, Notre Dame Journal of Formal Logic 26 (1985), 129-138, Proceedings of the 1980/1 Jerusalem Model Theory year.

[Sh:199] Saharon Shelah, Remarks in abstract model theory, Annals of Pure and Applied Logic 29 (1985), 255-288. 